\title{
Dangerous unmarked genes
}

Loss of imprinting (LOI) at specific loci has been implicated in several cases of tumorigenesis, probably as a result of imbalanced expression of potential imprinted tumour-suppressor genes and oncogenes. Previous studies have demonstrated that LOI is associated with tumorigenesis, not that it causes it. The consequences of a global LOI have not been addressed either, merely the consequences of single-gene LOI or imbalanced imprinting (having an entirely maternally or paternally imprinted genome). Now, Rudolf Jaenisch and colleagues have demonstrated that global LOI leads to tumour formation.

They used conditional mutants of DNA methyltransferase to transiently remove methylation from mouse embryonic stem (ES) cells. When methylation was restored, imprinting patterns were lost - the maternal and paternal genomes were no longer differentially methylated. The authors derived fibroblasts from these ES cells and found that they were immortalized, grew at an increased rate and resisted inhibition by transforming growth factor $\beta$, a cytokine that inhibits the growth of various cell types.

Several tumour-suppressor genes, such as $\operatorname{Igf2r}$, Tsp 1 and $p 57$, were underexpressed in the fibroblasts, and oncogenes, such as Peg3, Peg5 and $I g f 2$ were overexpressed. When the non-imprinted fibroblasts were injected into immunodeficient mice there was some tumorigenesis, compared with none in the controls. However, when the fibroblasts were also transfected with constitutively active Ras, tumorigenesis was much faster. The authors suggest that this is because Ras and LOI cooperate to form tumours.

Chimeric mice that were created from a mixture of non-imprinted and normal ES cells all had tumours by 18 months of age, in contrast to the controls in which there was only one case of tumour formation. All the tumours in the chimeric mice were derived from the non-imprinted cells. Importantly, tumours were not seen in the offspring of the chimaeras, as imprinting is reset during gametogenesis.

Imprinting is therefore an epigenetic tumour-suppressing phenomenon. When imprinting is lost, cells are immortalized through the inappropriate regulation of both tumour suppressors and oncogenes. Further genetic alterations are then required for full transformation.

Patrick Goymer

6) References and links

ORIGINAL RESEARCH PAPER Holm, T. M. et al. Global loss of imprinting leads to widespread tumorigenesis in adult mice. Cancer Cell 8, 275285 (2005)

FURTHER READING Robertson, K. D. DNA methylation and human disease. Nature Rev. Genet. 6, 597-610 (2005) | Ushijima, T. Detection and interpretation of altered methylation patterns in cancer cells. Nature Rev. Cancer 5, 223-231 (2005)

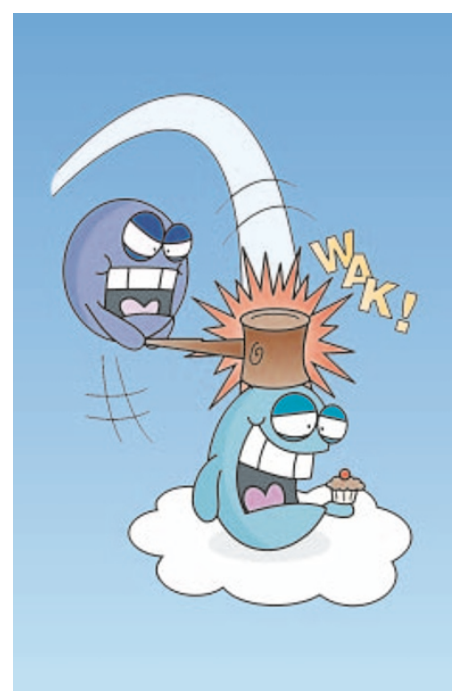

